\title{
Spotlight on North Carolina
}

January 2018

This brief highlights the major strategies, lessons learned, and outcomes from North Carolina's experience during the quality demonstration funded by the Centers for Medicare \& Medicaid Services (CMS) through the Children's Health Insurance Program Reauthorization Act of 2009 (CHIPRA) from February 2010 to February 2016. In this demonstration, CMS awarded 10 grants that supported efforts in 18 States to identify effective, replicable strategies for enhancing the quality of health care for children. With funding from CMS, the Agency for Healthcare Research and Quality (AHRQ) led the evaluation of the program.

\section{North Carolina used quality measures to drive quality improvement}

In collaboration with Community Care of North Carolina (CCNC), a public-private partnership covering all 100 counties in the State, North Carolina expanded the scope of its collection, reporting, and use of the Core Set of Children's Health Care Quality Measures for Medicaid and CHIP (Child Core Set). ${ }^{1}$ Using CHIPRA quality demonstration funds, North Carolina-

- Reported 25 Child Core Set measures to CMS by the end of the grant period, up from 2 in 2010. North Carolina drew on data from various State agencies to calculate and report the measures to CMS. It also hired an independent vendor to collect survey data on patient experience.

- Improved existing practice-level quality reports. North Carolina incorporated additional child-focused measures, including Child Core Set measures, into quarterly reports that the State makes available to all practices serving Medicaid and CHIP beneficiaries. In a 2014 survey of North Carolina pediatricians and family physicians, 58 percent of respondents reported that they received quality reports with selected Child Core Set measures included. Seventythree percent felt that the quality reports were effective for improving the quality of care for children, but only 33 percent said that they had started to use the quality reports to improve the quality of care for children. ${ }^{2}$
North Carolina's Goals: Improve quality of care for children by- Calculating, reporting, and using quality measures.

- Helping practices strengthen the medical home model for children with special health care needs.

- Testing the Children's Electronic Health Record Format.

- Helped more than 200 practices improve care quality. North Carolina hired 14 pediatric quality improvement (QI) specialists-one for each CCNC provider networkto analyze network- and practice-level data and work with practices to set QI goals. QI specialists helped practices develop QI teams, identify QI activities, and improve targeted care processes. When the State determined that some QI specialists needed additional QI skills to be most effective, North Carolina hired a statewide QI coordinator and invested in substantial training in technical QI and clinical content areas to ensure that the specialists were prepared to support practices across a range of activities. North Carolina also established a workgroup, consisting of QI specialists, champions for children's health care, and care managers from all CCNC provider networks. The work group met monthly to discuss priorities for clinical QI activities.

- Improved performance on quality measures. North Carolina made modest but meaningful improvements on several quality measures (Figure 1) during a 15-month period. Demonstration staff believe that collaboration between the QI specialists and practices contributed to the changes.

\section{Practices improved delivery of recommended preventive services through use of the medical home model}

North Carolina used a learning collaborative model to educate 23 practices on strengthening their medical home characteristics for children, especially children with special health care needs (CSHCN). Participating practices- 
Figure 1. Increases in measures for all children enrolled in Medicaid and CHIP in North Carolina

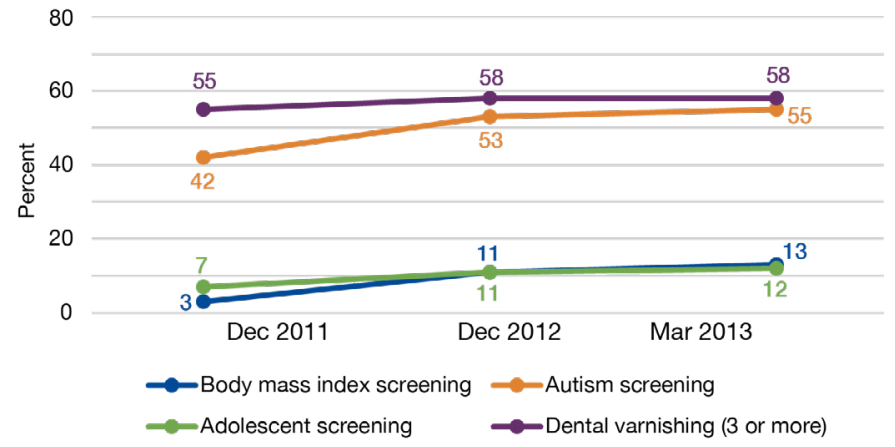

Note: Data were reported by North Carolina and not independently validated.

- Built their QI capacity. Participating practices developed QI teams charged with improving the practices' quality of care. The teams attended group in-person and virtual learning sessions delivered by the State and received individualized assistance. Clinicians were eligible to receive Maintenance of Certification credit for completing training modules. Initially, the State encountered challenges both in maintaining practice participation in learning activities and motivating practices to use data to drive QI. In response, the State offered practices: (1) financial incentives tied to participation in learning activities and (2) individualized assistance not only to help practice-level QI teams run and use data reports from their electronic health records (EHRs) but also to implement QI activities. The State also developed a video series and clinical toolkit to help practices engage adolescents in their own care. ${ }^{3}$

- Implemented care process improvements. Participating practices improved the process for delivering preventive care in several ways, particularly for CSHCN (Figure 2). Many practices instituted: (1) the use of validated, State-recommended mental health and developmental screening questionnaires, (2) regular measurement and recording of children's body mass index, (3) the use of motivational interviewing to help families improve nutrition and increase physical activity among children at risk of obesity, and (4) the use of dental fluoride varnish for children. Practices reported high levels of developmental screening for infants and young children at baseline (90 percent or greater), and they sustained this level of care after the collaborative ended.
Figure 2. Examples of reported Ql among practices participating in the second North Carolina CHIPRA-funded learning collaborative

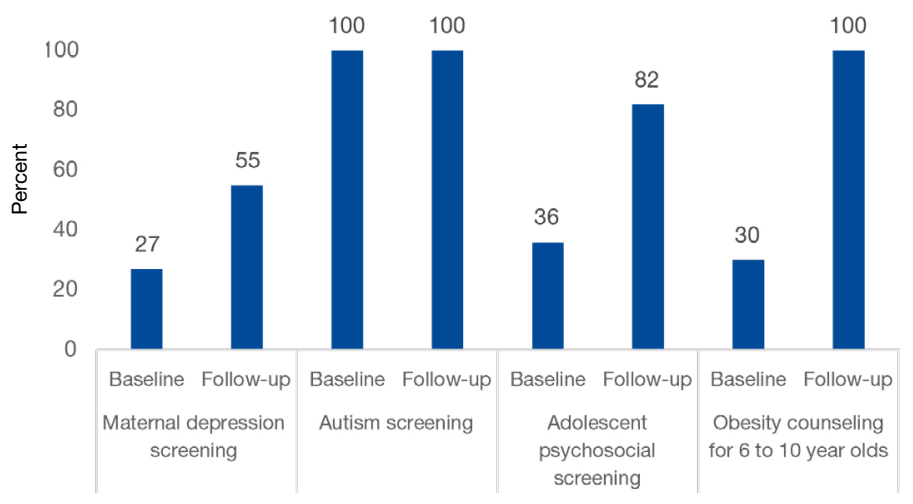

Note: Data were reported by North Carolina and not independently validated. The baseline was June 2012 with follow-up in June 2014.

\section{Practices used new EHR features for improved capture of information about children}

The Model Children's EHR Format (Format) is a set of recommended requirements for EHR data elements, data standards, and functionality released by the United States Department of Health and Human Services in February 2013. ${ }^{4}$ To assess the Format's usefulness, North Carolina worked concurrently with national EHR vendors and State-level child-serving practices that used the vendors' products. With support from the demonstration, the State hired four coaches to help the practices participate in the assessment. The State-

- Helped more than 25 practices use their EHRs more effectively. The EHR coaches surveyed practices and vendors to understand how practices' existing EHR functionality compared with the Format. Coaches and vendors then helped practices use existing features that met Format requirements. For example, coaches helped practices access tools that enabled direct entry of data from screening questionnaires into EHRs.

- Encouraged vendors to develop new functionalities and provide training. To drive changes in EHR functionality that met Format requirements, State demonstration staff developed specifications to guide vendors in making modifications to their existing EHRs. As a result, some vendors enhanced their systems' reporting capabilities; others produced tools to capture data at the point of care 
and created report views to assist practices in population management and QI. While some vendors made changes, the State indicated that working with them was a slow and difficult process. Some vendors were reluctant to add functionalities given competing demands, including meeting meaningful use requirements, and concerns that project participation might adversely affect their competitive edge.

\section{Key demonstration takeaways}

- By improving practice-level quality reporting and facilitating QI, the State supported modest improvements to statewide rates of routine adolescent, autism, and obesity screening, and the provision of dental varnishing.

- North Carolina leveraged existing infrastructure to implement a statewide model of QI coaching. QI specialists trained practices to use quality measure reports to identify priorities, conduct QI activities, track progress, and standardize processes to improve the delivery of preventive services.

- Practices participating in the learning collaborative reported enhanced QI capacity and implementation of new care processes.

- Although some EHR vendors improved their systems to conform to the Format, many were slow to build Format requirements into their products. In the interim, providing direct assistance to practices helped improve their use of EHR functionality.

\section{Endnotes}

1. For more information on the Child Core Set, visit https:/ / www. medicaid.gov / medicaid / quality-of-care / downloads / 2016-child-coreset.pdf.

2. For more information about the survey, visit http: / / www. academicpedsjnl.net/article/S1876-2859(16)30364-3/ abstract. We conducted a cross-sectional survey of pediatricians and family physicians that provide primary care to publicly insured children in North Carolina. The final sample included responses from 235 clinicians (46.9 percent response rate). Survey weights were used to calculate univariate statistics.

3. The Engaging Adolescents Video Series is available at vimeopro.com/ emergentpictures/engaging-adolescents.

4. For more information on the Format, visit https: / /www.ahrq.gov/ policymakers/chipra/ehrformatfaq.html.

\section{Continuing Efforts in North Carolina}

After North Carolina's CHIPRA quality demonstration ended in February 2016-

- CCNC expected to integrate the tracking of most Child Core Set measures into its ongoing activities as part of the State's Medicaid performance measurement.

- CCNC expected to integrate the quarterly reporting of practicelevel quality measures into its ongoing quality strategy.

- CCNC planned to continue employing QI specialists in the networks to work with practices that serve children and to start using QI specialists to improve care for adults.

- CCNC expected to integrate local health coordinators into network QI teams to help promote medical home activities in pediatric practices.

- The State anticipated disseminating QI materials developed during the CHIPRA quality demonstration to child-serving practices. The materials were expected to include guides on motivational interviewing for obesity and shared decisionmaking for asthma and a video series and clinical toolkit to help practices engage adolescents in their own care.

- The State planned to collaborate with the North Carolina Pediatric Society to operate the Fostering Health North Carolina project. This included continuing to offer training for childserving practices on the medical home model and appropriate care for children in foster care.

- Practices that participated in the learning collaboratives expected to offer new care processes developed under the CHIPRA quality demonstration to all children, not just those insured by Medicaid/CHIP.

- The State planned to seek additional funding to continue working with practices and engaging vendors to improve EHR functionality for children. 


\section{LEARN MORE}

North Carolina's CHIPRA quality demonstration experiences are described in more detail on the national evaluation Web site at http://www.ahrq.gov/policymakers/chipra/demoeval/demostates/nc.html.

The following products highlight North Carolina's experiences-

- Evaluation Highlight No. 1: Four States' approaches to practice-level quality measurement and reporting.

- Evaluation Highlight No. 2: How are States and evaluators measuring medical homeness in the CHIPRA Quality Demonstration Grant Program?

- Evaluation Highlight No. 3: How are CHIPRA quality demonstration States working to improve adolescent health?

- Evaluation Highlight No. 10: How are CHIPRA quality demonstration States testing the Children's Electronic Health Record Format?

- Evaluation Highlight 11: How are CHIPRA quality demonstration States using quality reports to drive health care improvements for children?

- Evaluation Highlight No. 12: How are CHIPRA quality demonstration States improving perinatal care?

- Evaluation Highlight No. 13: How did CHIPRA quality demonstration States employ learning collaboratives to improve children's health care quality?

- Article: Devers K, Foster L, Brach C. Nine states' use of collaboratives to improve children's health care quality in Medicaid and CHIP. Acad Pediatr 2013;13(6):S95-102. PMID: 24268093.

- Article: Zickafoose J, Ireys H, Swinburn A, Simpson L. Primary care physicians' experiences with and attitudes toward pediatric quality reporting. Acad Pediatr 2016;16(8):750-759.
The information in this brief draws on interviews conducted with staff in North Carolina agencies and participating practices, a survey of child-serving providers, and a review of project reports submitted by North Carolina to CMS.

The following staff from Mathematica Policy Research and the Urban Institute contributed to data collection or the development of this summary: Dana Petersen, Mynti Hossain, Rachel Burton, and Christal Ramos. 